\title{
ANALYTIC FUNCTIONS OF NON-BAZILEVIČ TYPE AND STARLIKENESS
}

\section{MILUTIN OBRADOVIĆ and SHIGEYOSHI OWA}

(Received 23 October 2000)

\begin{abstract}
Two classes $\overline{\mathscr{P}}_{n}(\mu, \alpha, \lambda)$ and $\overline{\mathscr{P}}_{n}(\mu, \alpha, \lambda)$ of analytic functions which are not Bazilevič type in the open unit disk $\mathbb{U}$ are introduced. The object of the present paper is to consider the starlikeness of functions belonging to the classes $\overline{\mathscr{P}}_{n}(\mu, \alpha, \lambda)$ and $\overline{\mathscr{P}}_{n}(\mu, \alpha, \lambda)$.

2000 Mathematics Subject Classification. 30C45.
\end{abstract}

1. Introduction. Let $\mathbb{U}=\{z \in \mathbb{C}:|z|<1\}$ denote the open unit disk in the complex plane $\mathbb{C}$. For $n \geqq 1$, we define

$$
\mathscr{A}_{n}=\left\{f: f(z)=z+\sum_{n+1}^{\infty} a_{j} z^{j} \text { analytic in } \mathbb{U}\right\} .
$$

Also, we need the following notations and definitions. Let

$$
\mathscr{S}^{*}=\left\{f \in \mathscr{A}_{1}: \operatorname{Re}\left\{\frac{z f^{\prime}(z)}{f(z)}\right\}>0, z \in \mathbb{U}\right\}
$$

be the class of starlike functions (with respect to the origin) in $\mathbb{U}$, and let

$$
\mathscr{T}_{\lambda}=\left\{f \in \mathscr{A}_{1}:\left|\frac{z f^{\prime}(z)}{f(z)}-1\right|<\lambda, 0<\lambda \leqq 1, z \in \mathbb{U}\right\}
$$

be the subclass of $\mathscr{S}^{*}$. Further we define

$$
\mathscr{B}(\mu, \lambda)=\left\{f \in \mathscr{A}_{1}:\left|f^{\prime}(z)\left(\frac{f(z)}{z}\right)^{\mu-1}-1\right|<\lambda, \mu>0,0<\lambda \leqq 1, z \in \mathbb{U}\right\}
$$

which is the subclass of Bazilevič class of univalent functions (cf. [1]). Ponnusamy [8] has considered the starlikeness and other properties of functions $f(z)$ in the class $\mathscr{B}(\mu, \lambda)$. For negative $\mu$, that is, for $-1<\mu<0$, which is better to write (with $0<\mu<1$ ) in the form

$$
\overline{\mathscr{B}}(\mu, \lambda)=\left\{f \in \mathscr{A}_{1}:\left|f^{\prime}(z)\left(\frac{z}{f(z)}\right)^{1+\mu}-1\right|<\lambda, 0<\mu<1,0<\lambda<1, z \in \mathbb{U}\right\},
$$

we obtain the class which was considered earlier by Obradović [3, 4], Obradović and Owa [5], and Obradović and Tuneski [6].

For the limit case $\mu=0$, this class becomes the class $\mathcal{T}_{\lambda}$. When $\mu=1$, this class 
becomes the class of univalent functions $f(z)$ satisfying

$$
\left|\frac{z^{2} f^{\prime}(z)}{f(z)^{2}}-1\right|<\lambda
$$

which was studied by Ozaki and Nunokawa [7].

Next, for $\alpha=\left(\alpha_{1}, \alpha_{2}, \ldots, \alpha_{k}\right) \in \mathbb{R}^{k}$ with $\alpha_{j} \in \mathbb{R}$, we define the operator $D_{\alpha}$ by

$$
D_{\alpha}=1+\alpha_{1} z \frac{d}{d z}+\alpha_{2} z^{2} \frac{d^{2}}{d z^{2}}+\cdots+\alpha_{k} z^{k} \frac{d^{k}}{d z^{k}},
$$

and, by virtue of the operator $D_{\alpha}$, the subclasses

$$
\begin{aligned}
& \overline{\mathscr{P}}_{n}(\mu, \alpha, \lambda)=\left\{f \in \mathscr{A}_{n}:\left|D_{\alpha}\left(f^{\prime}(z)\left(\frac{z}{f(z)}\right)^{1+\mu}\right)-1\right|<\lambda, 0<\mu<1,0<\lambda<1, z \in \mathbb{U}\right\}, \\
& \overline{\mathscr{P}}_{n}(\mu, \alpha, \lambda)=\left\{f \in \mathscr{A}_{n}:\left|D_{\alpha}\left[\left(f^{\prime}(z)\left(\frac{z}{f(z)}\right)^{1+\mu}\right)^{\prime}\right]\right|<\lambda, 0<\mu<1,0<\lambda<1, z \in \mathbb{U}\right\}
\end{aligned}
$$

of $\mathscr{A}_{n}$.

Samaris [9] has investigated the appropriate classes for the case (1.4), and has obtained results which are stronger than those given earlier and in several cases sharp ones. By using the method by Samaris [9], we will generalize some results given in $[3,4]$, and we will obtain some new results. We also note that we cannot directly apply some nice estimates given by Samaris [9].

For $\alpha=\left(\alpha_{1}, \alpha_{2}, \ldots, \alpha_{k}\right) \in \mathbb{R}^{k}$, we define the polynomial $P_{\alpha}(x)$ by

$$
P_{\alpha}(x)=1+\alpha_{1} x+\alpha_{2} x(x-1)+\cdots+\alpha_{k} x(x-1) \cdots(x-k+1) .
$$

In this paper, we will use the classes such that $\alpha=0\left(P_{\alpha}(x)=1\right)$ or $P_{\alpha}(x)$ has nonpositive real zeros given by $\rho_{j}(j=1,2,3, \ldots, k)$. In this case, we can write

$$
P_{\alpha}(x)=\alpha_{k} \prod_{j=1}^{k}\left(x-\rho_{j}\right)
$$

with

$$
\alpha_{k} \prod_{j=1}^{k}\left(-\rho_{j}\right)=1 .
$$

Further, for $t=\left(t_{1}, t_{2}, \ldots, t_{k}\right) \in(0,1)^{k}$ such that $t_{j} \in(0,1)$, we denote $t_{\alpha}$ by

$$
t_{\alpha}=t_{1}^{-1 / \rho_{1}} t_{2}^{-1 / \rho_{2}} \cdots t_{k}^{-1 / \rho_{k}}=\prod_{j=1}^{k} t_{j}^{-1 / \rho_{j}} .
$$

If $P_{\alpha}(x)=1$, then we define $t_{\alpha}=1$. Also if $n=1,2,3, \ldots$, then we denote by $\mathscr{W}_{\alpha}$ the class of analytic functions $w(z)$ in $\mathbb{U}$ for which $|w(z)| \leqq|z|^{n}(z \in \mathbb{U})$.

2. Starlikeness of the classes $\overline{\mathscr{P}}_{n}(\mu, \alpha, \lambda)$ and $\overline{\mathscr{P}}_{n}(\mu, \alpha, \lambda)$. Our first result is contained in the following theorem. 
THEOREM 2.1. Let $\overline{\mathscr{B}}_{n}(\mu, \alpha, \lambda)$ be the class defined by (1.8) for which $(n-\mu) P_{\alpha}(n)-$ $\lambda n>0(n=1,2,3, \ldots), 0<\mu<1$.

(i) If $\lambda n /\left((n-\mu) P_{\alpha}(n)-\lambda \mu\right) \leqq r$, then $\overline{\mathscr{P}}_{n}(\mu, \alpha, \lambda) \subset \mathscr{T}_{r}$,

(ii) if $\lambda \leqq P_{\alpha}(n)((n-\mu) /(n+\mu))$, then $\overline{\mathscr{P}}_{n}(\mu, \alpha, \lambda) \subset \mathscr{T}_{1}$.

Proof. From

$$
D_{\alpha}\left(f^{\prime}(z)\left(\frac{z}{f(z)}\right)^{1+\mu}\right)=1+\lambda w(z)=1+\lambda \sum_{n=1}^{\infty} w_{n} z^{n} \quad\left(w \in W_{n}\right),
$$

we obtain

$$
\begin{aligned}
& f^{\prime}(z)\left(\frac{z}{f(z)}\right)^{1+\mu}=1+\lambda \sum_{n=1}^{\infty} w_{n} \frac{z^{n}}{P_{\alpha}(n)} \\
& \left(\frac{z}{f(z)}\right)^{\mu}=1-\lambda \sum_{n=1}^{\infty} w_{n}\left(\frac{\mu}{n-\mu}\right) \frac{z^{n}}{P_{\alpha}(n)} .
\end{aligned}
$$

Since

$$
\frac{1}{P_{\alpha}(n)}=\int_{[0,1]^{k}} t_{\alpha}^{n}, \quad \frac{\mu}{P_{\alpha}(n)(n-\mu)}=\int_{[0,1]^{k+1}} t_{\alpha}^{n} t_{k+1}^{n / \mu-2},
$$

using (2.2), we have

$$
\begin{aligned}
& f^{\prime}(z)\left(\frac{z}{f(z)}\right)^{1+\mu}=1+\lambda \int_{[0,1]^{k}} w\left(t_{\alpha} z\right), \\
& \left(\frac{z}{f(z)}\right)^{\mu}=1-\lambda \int_{[0,1]^{k+1}} w\left(t_{\alpha} t_{k+1}^{1 / \mu} z\right) t_{k+1}^{-2} .
\end{aligned}
$$

From (2.4), we easily obtain that

$$
\frac{z f^{\prime}(z)}{f(z)}=\frac{1+\lambda \int_{[0,1]^{k}} w\left(t_{\alpha} z\right)}{1-\lambda \int_{[0,1]^{k+1}} w\left(t_{\alpha} t_{k+1}^{1 / \mu} z\right) t_{k+1}^{-2}}
$$

and from here

$$
\begin{aligned}
\left|\frac{z f^{\prime}(z)}{f(z)}-1\right| & \leqq \lambda \frac{\int_{[0,1]^{k}}\left|w\left(t_{\alpha} z\right)\right|+\int_{[0,1]^{k+1}}\left|w\left(t_{\alpha} t_{k+1}^{1 / \mu} z\right)\right| t_{k+1}^{-2}}{1-\lambda \int_{[0,1]^{k+1}}\left|w\left(t_{\alpha} t_{k+1}^{1 / \mu} z\right)\right| t_{k+1}^{-2}} \\
& <\lambda \frac{\int_{[0,1]^{k}} t_{\alpha}^{n}+\int_{[0,1]^{k+1}} t_{\alpha}^{n} t_{k+1}^{n / \mu-2}}{1-\lambda \int_{[0,1]^{k+1}} t_{\alpha}^{n} t_{k+1}^{n / \mu-2}}=\frac{\lambda n}{(n-\mu) P_{\alpha}(n)-\lambda \mu},
\end{aligned}
$$

from which the conclusion of the theorem easily follows.

REMARK 2.2. For $n=1$ and $P_{\alpha}=1$ from Theorem 2.1, we have the result given by Obradović [3].

REMARK 2.3. From (2.5), similar to the proof of Theorem 2.1, for the class $\overline{\mathscr{B}}_{n}(\mu, \alpha, \lambda)$ we have

$$
\left|\frac{z f^{\prime}(z)}{f(z)}-t\right| \leqq|1-t|+\frac{\lambda n}{(n-\mu) P_{\alpha}(n)-\lambda \mu} \quad(t>0) .
$$


If $\lambda_{1}=\lambda n /\left((n-\mu) P_{\alpha}(n)-\lambda \mu\right)<1$ and $t \geqq\left(1+\lambda_{1}\right) / 2$, then we have

$$
\overline{\mathscr{P}}_{n}(\mu, \alpha, \lambda) \subset\left(\mathscr{Y}^{*}\right)_{t},
$$

where

$$
\left(\mathscr{S}^{*}\right)_{t}=\left\{f \in \mathscr{A}_{1}:\left|\frac{z f^{\prime}(z)}{f(z)}-t\right|<t, z \in \mathbb{U}\right\} .
$$

Especially, for $n=1$ and $\alpha=0$, we have

$$
\overline{\mathscr{B}}(\mu, \lambda) \subset\left(\mathscr{S}^{*}\right)_{t}
$$

for $\lambda /(1-\mu-\lambda)<1$ and $t \geqq 1 / 2+\lambda /(2(1-\mu-\lambda))$.

In the next theorem for the class $\overline{\mathscr{B}}(\mu, \lambda) \cap \mathscr{A}_{n}=\overline{\mathscr{B}}_{n}(\mu, 0, \lambda)$, we will prove that the appropriate results are the best possible.

THEOREM 2.4. Let $\overline{\mathscr{B}}(\mu, \lambda) \cap A_{n}$ be the class for which $n-\mu-\lambda \mu>0$. Then

(i) $\overline{\mathscr{B}}(\mu, \lambda) \cap \mathscr{A}_{n} \subset \mathscr{T}_{r}$ if and only if $\lambda n /(n-\mu-\lambda \mu) \leqq r$ and

(ii) $\overline{\mathscr{B}}(\mu, \lambda) \cap \mathscr{A}_{n} \subset \mathscr{S}^{*}$ if and only if $\lambda \leqq(n-\mu) / \sqrt{(n-\mu)^{2}+\mu^{2}}$.

Proof. For $t_{1} \in \mathbb{R}$ and $t_{2} \in \mathbb{R}$, with the lemma by Fournier [2], there exists a sequence of functions $\phi_{k}(z)$ analytic in the closed unit disk $\mathbb{U}$ such that $\left|\phi_{k}(z)\right| \leqq|z|$, $\lim _{k \rightarrow \infty} \phi_{k}(z)=z e^{\mathrm{it}}{ }_{1}, \lim _{k \rightarrow \infty} \phi_{k}(1)=e^{\mathrm{it} 2}$ uniformly on compact subsets of $\mathbb{U}$. If $w_{k}(z)=$ $z^{n-1} \phi_{k}(z)$, then we consider the sequence $f_{k}(z) \in \overline{\mathscr{B}}(\mu, \lambda) \cap \mathscr{A}_{n}$ which is given by

$$
f_{k}^{\prime}(z)\left(\frac{z}{f_{k}(z)}\right)^{1+\mu}=1+\lambda w_{k}(z)
$$

or

$$
\frac{z f_{k}^{\prime}(z)}{f_{k}(z)}=\frac{1+\lambda w_{k}(z)}{1-\lambda \int_{0}^{1} w_{k}\left(t^{1 / \mu} z\right) t^{-2} d t} .
$$

Since $\lim _{k \rightarrow \infty} w_{k}(z)=z^{n} e^{\mathrm{it}_{1}}$ and $\lim _{k \rightarrow \infty} w_{k}(1)=e^{\mathrm{it} 2}$, we see that

$$
\lim _{k \rightarrow \infty} \lim _{z \rightarrow 1} \frac{z f_{k}^{\prime}(z)}{f_{k}(z)}=\frac{1+\lambda e^{\mathrm{it}_{1}}}{1-\lambda(\mu /(n-\mu)) e^{\mathrm{it}_{2}}} .
$$

For $t_{1}=0$ and $t_{2}=0$, we get

$$
\lim _{k \rightarrow \infty} \lim _{z \rightarrow 1}\left|\frac{z f_{k}^{\prime}(z)}{f_{k}(z)}-1\right|=\frac{\lambda n}{n-\mu-\lambda \mu} .
$$

Let $0<\lambda<1$ and $q_{1} \in[0, \pi / 2], q_{2} \in[0, \pi / 2]$ such that $\sin q_{1}=\lambda, \sin q_{2}=\lambda \mu /(n-\mu)$. If we choose $t_{1}=q_{1}+\pi / 2, t_{2}=\pi / 2-q_{2}$, then we obtain

$$
\arg \left(1+\lambda e^{\mathrm{it}_{1}}\right)=q_{1}, \quad \arg \left(1-\frac{\lambda \mu}{n-\mu} e^{\mathrm{it} 2}\right)=-q_{2},
$$

and therefore

$$
\lim _{k \rightarrow \infty} \lim _{z \rightarrow 1}\left|\arg \left(\frac{z f_{k}^{\prime}(z)}{f_{k}(z)}\right)\right|=q_{1}+q_{2} .
$$


From $\cos \left(q_{1}+q_{2}\right) \geqq 0$ or $\sin ^{2} q_{1}+\sin ^{2} q_{2}-1 \leqq 0$, we have the statement (ii) (one part) of the theorem. We note that the "if" part of the theorem follows from the result of Theorem 2.1 and from the result given by Obradović and Owa [5].

THEOREM 2.5. Let $\overline{\mathscr{P}}_{n}(\mu, \alpha, \lambda)$ be the class defined by (1.9) such that $n(n-\mu) P_{\alpha}(n-1)$ $-\lambda n>0(n=1,2,3, \ldots), 0<\mu<1$. If

$$
\frac{\lambda n}{n(n-\mu) P_{\alpha}(n-1)-\lambda \mu} \leqq r
$$

then

$$
\overline{\mathscr{P}}_{n}(\mu, \alpha, \lambda) \subset \mathscr{T}_{r} .
$$

Proof. The proof of this theorem is similar to that of Theorem 2.1. By virtue of

$$
D_{\alpha}\left\{\left(f^{\prime}(z)\left(\frac{z}{f(z)}\right)^{1+\mu}\right)^{\prime}\right\}=\lambda w(z) \quad\left(w \in W_{n-1}\right),
$$

we have

$$
\begin{gathered}
\left\{f^{\prime}(z)\left(\frac{z}{f(z)}\right)^{1+\mu}\right\}^{\prime}=\lambda \int_{[0,1]^{k}} w\left(t_{\alpha} z\right), \\
f^{\prime}(z)\left(\frac{z}{f(z)}\right)^{1+\mu}=1+\lambda \int_{[0,1]^{k+1}} w\left(t_{\alpha} t_{k+1} z\right) z .
\end{gathered}
$$

From the last relation, we see that

$$
\left(\frac{z}{f(z)}\right)^{\mu}=1-\lambda \int_{[0,1]^{k+2}} w\left(t_{\alpha} t_{k+1} t_{k+2}^{1 / \mu} z\right) t_{k+2}^{-2} z
$$

and so

$$
\frac{z f^{\prime}(z)}{f(z)}=\frac{1+\lambda \int_{[0,1]^{k+1}} w\left(t_{\alpha} t_{k+1} z\right) z}{1-\lambda \int_{[0,1]^{k+2}} w\left(t_{\alpha} t_{k+1} t_{k+2}^{1 / \mu} z\right) t_{k+2}^{-2} z} .
$$

By using (2.22), as in the proof of Theorem 2.1, we easily obtain that

$$
\left|\frac{z f^{\prime}(z)}{f(z)}-1\right| \leqq \frac{\lambda n}{n(n-\mu) P_{\alpha}(n-1)-\lambda \mu} .
$$

The statement of this theorem follows from the above inequality.

Finally we give the following example of the theorem.

EXAMPLE 2.6. For $P_{\alpha}=1+t$ and $0<\mu<1,0<\lambda<1$, if $f(z) \in \mathscr{A}_{n}$ satisfies

$$
\left|\left(f^{\prime}(z)\left(\frac{z}{f(z)}\right)^{1+\mu}\right)^{\prime}+z\left(f^{\prime}(z)\left(\frac{z}{f(z)}\right)^{1+\mu}\right)^{\prime \prime}\right|<\lambda
$$

implies that

$$
\left|\frac{z f^{\prime}(z)}{f(z)}-1\right| \leqq \frac{\lambda n}{n^{2}(n-\mu)-\lambda \mu}
$$




\section{REFERENCES}

[1] I. E. Bazilevič, Ueber einen Fall der Integrierbarkeit der Loewner-Kufarevschen Gleichungen durch Quadraturen [On a case of integrability in quadratures of the LoewnerKufarev equation], Mat. Sb. (N.S.) 37(79) (1955), 471-476 (Russian). MR 17,356e. Zbl 065.31002.

[2] R. Fournier, On integrals of bounded analytic functions in the closed unit disc, Complex Variables Theory Appl. 11 (1989), no. 2, 125-133. MR 90f:30014. Zbl 639.30016.

[3] M. Obradović, A class of univalent functions, Hokkaido Math. J. 27 (1998), no. 2, 329-335. MR 2000i:30028. Zbl 908.30009.

[4] _ A class of univalent functions. II, Hokkaido Math. J. 28 (1999), no. 3, 557-562. MR 2000i:30029. Zbl 938.30008.

[5] M. Obradović and S. Owa, Some sufficient conditions for strongly starlikeness, Int. J. Math. Math. Sci. 24 (2000), no. 9, 643-647. CMP 1793 994. Zbl 992.14925.

[6] M. Obradović and N. Tuneski, On certain properties of a class of univalent functions, Filomat (1999), no. 13, 59-65. CMP 1803013.

[7] S. Ozaki and M. Nunokawa, The Schwarzian derivative and univalent functions, Proc. Amer. Math. Soc. 33 (1972), 392-394. MR 45\#8821. Zbl 233.30011.

[8] S. Ponnusamy, Pólya-Schoenberg conjecture for Carathéodory functions, J. London Math. Soc. (2) 51 (1995), no. 1, 93-104. MR 96b:30036. Zbl 814.30017.

[9] N. Samaris, Differential inequalities and starlike functions, in preparation.

Milutin ObRadović: DePaRtment of Mathematics, Faculty of Technology and MetALluRgy, UNIVERSITY OF Belgrade, 4 KARNEGIJEVA STREET, 11000 BElgrade, YUgOSLAVIA

E-mail address: obrad@e1ab.tmf.bg.ac.yu

Shigeyoshi OWA: Department of Mathematics, Kinki University, Higashi-OSAKa, OSAKA 577-8502, JAPAN

E-mail address: owa@math.kindai .ac.jp 


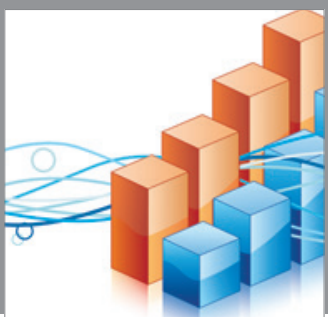

Advances in

Operations Research

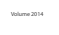

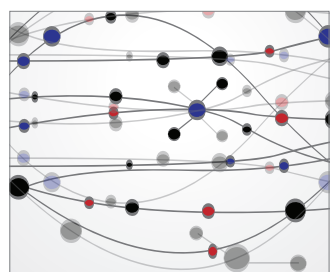

\section{The Scientific} World Journal
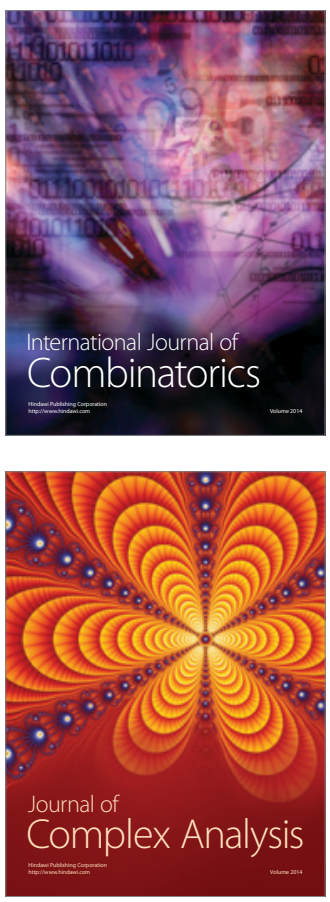

International Journal of

Mathematics and

Mathematical

Sciences
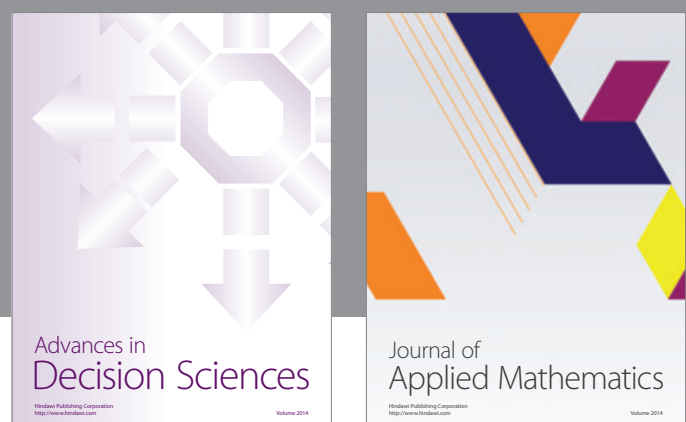

Journal of

Applied Mathematics
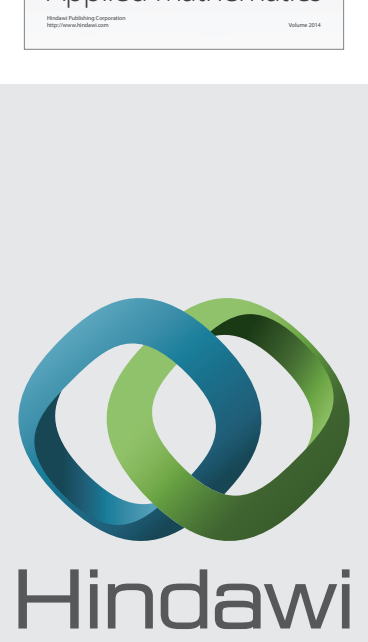

Submit your manuscripts at http://www.hindawi.com
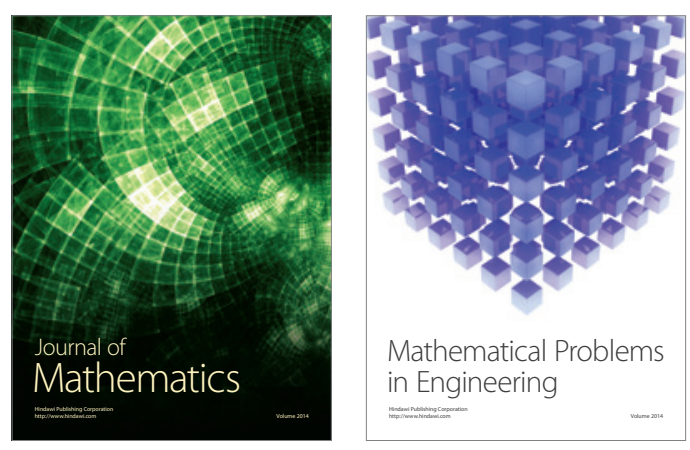

Mathematical Problems in Engineering
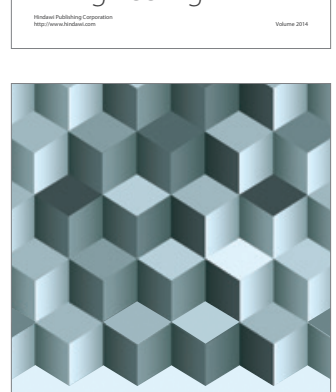

Journal of

Function Spaces
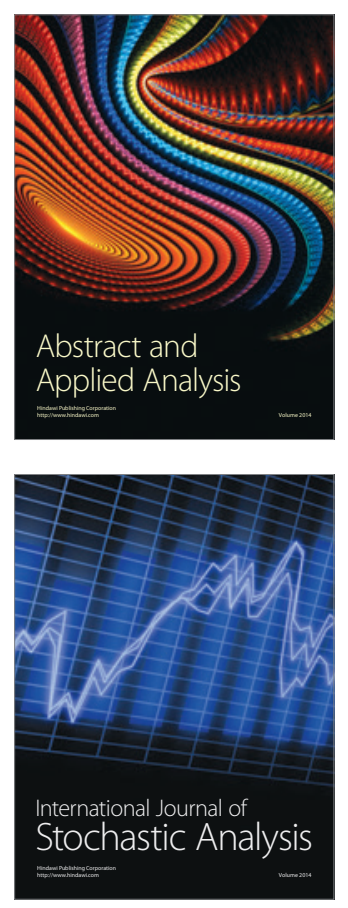

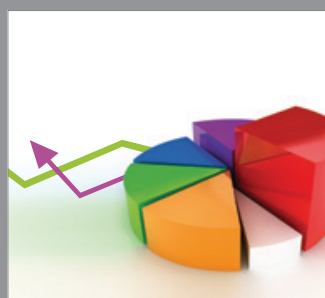

ournal of

Probability and Statistics

Promensencen
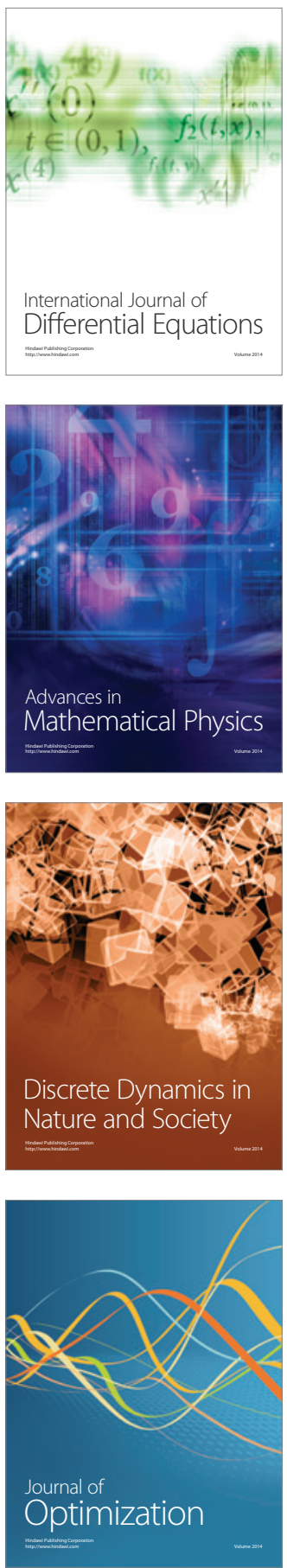